\title{
Experiencias pedagógicas en el ámbito del diseño para la transición. Estudio de un caso: El proyecto de comunicación del Espai Quiró
}

\begin{abstract}
Resumen
Cada vez más se espera de la disciplina del diseño que asuma un papel destacado como agente de transformación social en la transición hacia una sociedad sostenible. Estas demandas que provienen tanto del ámbito académico como social evidencian la necesidad de establecer sinergias de trabajo con los movimientos resilientes de las comunidades locales, lo cual plantea un fértil escenario emergente de nuevos retos para la pedagogía del diseño.
\end{abstract}

Por otra parte, desde el punto de vista metodológico, el encaje con estos movimientos de resiliencia social exige un proceso de diseño abierto y participativo que debe incluir a las comunidades en un sistema horizontal de toma de decisiones y al mismo tiempo ofrecer soluciones de diseño abiertas que puedan crecer y adaptarse siguiendo la evolución de las necesidades cambiantes de esas comunidades.

Dentro de estas premisas, estudiaremos el caso de un proyecto académico realizado en colaboración entre un grupo de estudiantes de diseño de la Escuela Superior de Diseño y Artes Plásticas y el colectivo autogestionado que dinamiza el proyecto de agricultura urbana y comunidad orgánica Espai Quiró en el barrio de La Salut en Barcelona.

Tanto el trabajo de la comunidad gestora del espacio como la intervención de los alumnos participantes en esta experiencia pedagógica respondían a un modelo de diseño comprometido con la ciudadanía y las asociaciones vecinales dirigidas por los propios ciudadanos, quienes superaron los modelos de conducta dominantes y participaron de la idea de transición hacia una nueva forma alternativa de vivir en las ciudades: con ritmos más pausados y sostenibles, abogando por un trabajo colaborativo y un empoderamiento del tejido asociativo, y con una reapropiación del espacio público por parte de las comunidades locales.
Joan Morales Moras

Doctor en Diseño

Docente Escola d'Art i Superior de

Disseny Serra i Abella

Barcelona, España

Correo electrónico:

jmoral35@xtec.cat

(1) orcid.org/0000-0001-5357-1112

Google Scholar

Recibido: Noviembre 23 de 2017

Aprobado: Agosto 18 de 2018

Palabras clave:

Diseño para la transición, diseño social, diseño de comunicación, diseño participativo, codiseño, diseño abierto.

Revista KEPES Año 16 No. 19 enero-junio 2019, págs. 217-251 ISSN: 1794-7111(Impreso) ISSN: 2462-8115 (En línea) DOI: $10.17151 /$ kepes.2017.15.18.9 


\section{Pedagogical experiences in the field of transition design. A case study: espai quiró the communication project}

\begin{abstract}
More and more the discipline of Design is expected to assume a prominent role as an agent for social transformation in the transition towards a sustainable society. These demands that come from both the academic and the social spheres, and show the need to establish new working synergies with the resilient movements of local communities which pose a fertile scenario emerging from new challenges for the pedagogy of design.
\end{abstract}

On the other hand, from a methodological point of view the fitting with these movements of social resilience requires an open and participatory design process, that must include communities in a horizontal decisionmaking system, and at the same time offer open design solutions that can grow and adapt following the evolution of the changing needs of these communities.

Within these premises, the case of a communications design project carried out in collaboration between a group of design students of Escola Superior de Disseny i Arts Plàstiques and the self-managed collective that dynamizes the project of urban agriculture and organic community named Espai Quiró in the neighborhood of la Salut (Barcelona) will be studied.

Both the work of the community managing the space and the intervention of the students participating in this pedagogical experience, responded to a model of design committed to citizenship and neighborhood associations, led by citizens themselves, that overcame the dominant models of behavior and participated in the idea of transition to a new alternative way of living in the cities: with a more unhurried and sustainable rhythm of life advocating collaborative work and empowerment of the associative network of the neighborhood and a re-appropriation of public space by local communities.
Key words:

Transition Design, Social Design, Communications Design, Participative Design, Co-Design, Open Design. 


\section{El papel del diseño para la transición y su enseñanza}

Ante la problemática que plantea el agotamiento del actual modelo consumista desde la economía de la transición se aboga por la necesidad de poner en marcha la fuerza creativa de aquellos dotados para proyectar una rebaja de nuestro nivel de consumo. Tal como apunta Rob Hopkins (2008): "liberando el genio colectivo de aquellos que nos rodean para diseñar creativa y proactivamente nuestro descenso energético, podemos construir formas de vida que estén más conectadas, sean más enriquecedoras y que reconozcan los límites biológicos de nuestro planeta" (p. 136). En este sentido existe un corpus de conocimiento interdisciplinar emergente que se propone asumir estos retos. Ese corpus de conocimiento, en palabras de Terry Irwin (2015), está "relacionado con la dinámica de cambio en sistemas complejos emergentes [...] y tiene el potencial para informar nuevos enfoques para el diseño y la resolución de problemas" (p. 234). De este modo, a partir de las distintas aproximaciones a los nuevos retos de la sociedad, el diseño para la transición se propone como un concepto catalizador que puede orientar a los nuevos diseñadores en este proceso de cambio. Así, para Irwin et al. (2015),

el concepto de diseño para la transición reconoce y parte de todos estos enfoques, aspira a actuar como un agente integrador entre ellos, y a educar a una generación de diseñadores calificados para trabajar en equipos transdisciplinarios desarrollando soluciones de transición. (p. 2)

Tal como se refleja, entre otros, en el "First Things First Manifesto 2000" por Barnbrook et al. (2000) —y en su versión original de 1964 — cada vez más se espera de la disciplina del diseño que asuma un papel destacado como agente de transformación en la transición hacia una sociedad sostenible. Estas demandas que provienen tanto del ámbito académico como social pueden encontrar en el diseño un elemento catalizador, formando diseñadores que

'La versión original de "First Things First manifesto" fue publicada por Ken Garland y otros 22 signatarios en Design, The Architects' Journal, SIA Journal, Ark, Modern Publicity y The Guardian en abril de 1964. 
trabajen en grupos interdisciplinares con el objetivo de ofrecer soluciones encaminadas a construir un modelo social más sostenible, lo cual plantea un fértil escenario de nuevos retos para la pedagogía del diseño.

Pero este cambio no es algo que pueda ni deba hacerse de manera vertical. La naturaleza y alcance de los retos evidencian la necesidad de establecer sinergias de trabajo con los movimientos resilientes de las comunidades locales, las cuales ofrecen soluciones a los problemas vecinales creando un tejido social comprometido con su barrio o ciudad. Seyfang y Smith (2007) defienden un modelo de innovaciones de base que describen como "redes innovadoras de activistas y organizaciones que lideran soluciones desde abajo hacia arriba para el desarrollo sostenible, soluciones que responden a la situación local y a los intereses y valores de las comunidades involucradas" (p. 585). Esto no es óbice para que estos proyectos locales puedan actuar como ensayos o modelos que se extiendan de forma viral o directamente como nodos de una red de transformación global.

En este sentido planteamos aquí una investigación-acción participativa en la que investigamos desde nuestra propia experiencia como participantes de una acción colectiva que busca el conocimiento a través del análisis de dicha experiencia. En este proceso colectivo de diseño y de aprendizaje se tomó en cuenta el saber popular de la comunidad vecinal con la que trabajamos en un proceso horizontal de toma de decisiones.

Así pues, como veremos más adelante, este planteamiento pedagógico incide en un sistema de codiseño participativo con soluciones abiertas cuyo fin es el trabajo con comunidades locales para promover el aprendizaje del diseño como agente de transformación social. 


\section{Estudio de caso. Proyecto de diseño de comunicación del Espai Quiró en el} barrio de La Salut

Como ejemplo de aplicación de estas premisas del diseño y de su enseñanza presentaremos a continuación el caso de una actividad académica de colaboración entre un grupo de alumnos de los "Estudios superiores de diseño gráfico" (ESDG) de la Escola Superior de Disseny i d'Arts Plàstiques (ESDAP) y el colectivo dinamizador del proyecto Espai Quiró. Para introducir esta actividad académica se presenta el proyecto vecinal del Espai Quiró y los principales colectivos implicados en él como son los alumnos que participaron en esta actividad.

\section{Presentación del proyecto Espai Quiró y los actores implicados}

\section{El proyecto Espai Quiró}

El proyecto Espai Quiró consiste en la autogestión por parte de un colectivo vecinal de los terrenos que ocupara la antigua Clínica Quirón en el barrio de La Salud de Barcelona (Figura 1). Se trata de un solar de $1914 \mathrm{~m}^{2}$ cuya entrada está en la avenida Mare de Deu de Montserrat 5-11 (Figura 2A) y que linda en su parte trasera con la calle Mare de Déu de la Salut (Figura 2B) y por los lados con un consultorio médico (Figura 2C) y un bloque de viviendas (Figura 2D). 


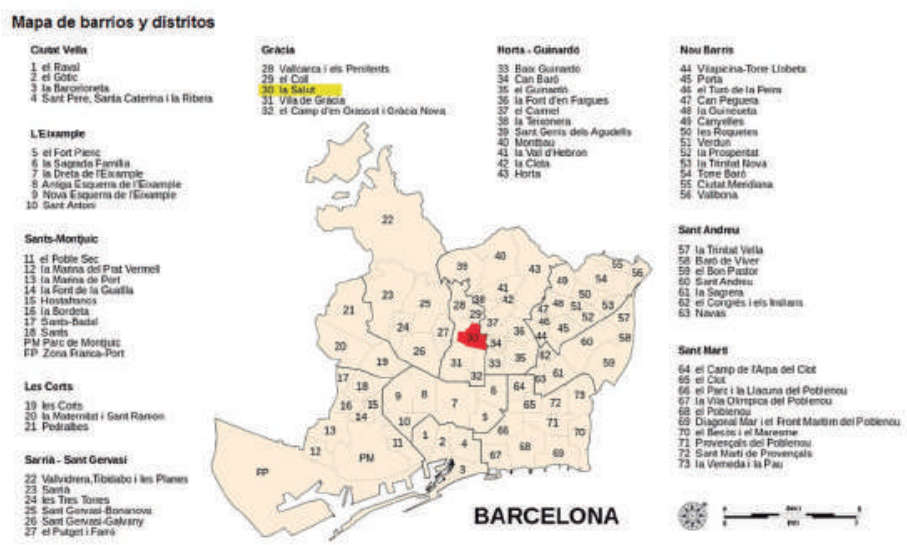

Figura 1. Ubicación del barrio La Salud en Barcelona. Fuente: Wikipedia.

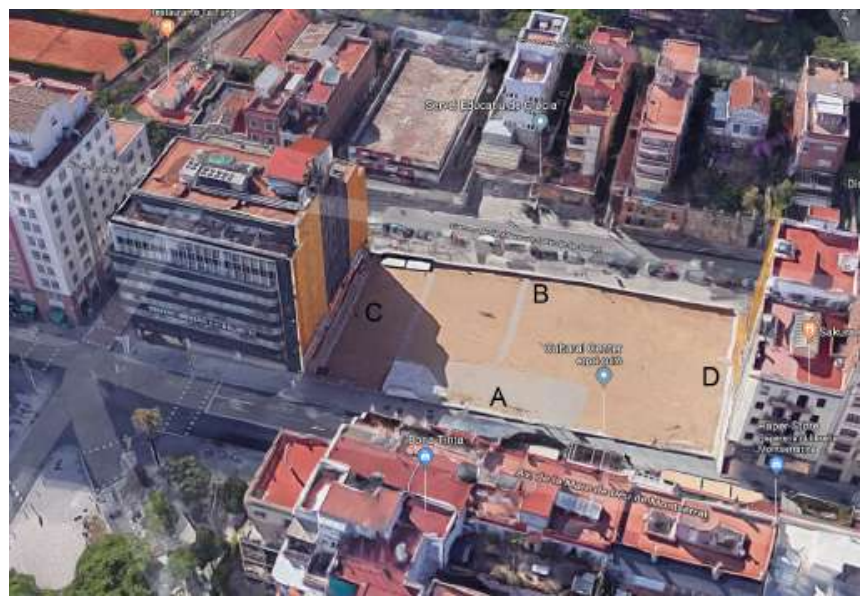

Figura 2. Vista cenital del solar que ocupara la antigua Clínica Quirón, tras la demolición del edificio. Fuente: Google Maps. 
Para entender la historia de este espacio y su gestión debemos remontarnos a 2007 cuando esta clínica cerró sus puertas para trasladarse a un nuevo edificio. Bien pronto hubo una primera acción vecinal que obtuvo una sentencia favorable para evitar la construcción de viviendas en este espacio destinado a servicios sanitarios. En 2012 el ayuntamiento adquirió la propiedad y tras derribar el edificio en 2014 anunció en enero de 2015 un nuevo plan de usos sociales para el solar previsto inicialmente para fines de 2016, y que a día de hoy sigue pendiente de ejecución. Ante la perspectiva probable de tener el espacio cerrado durante un período incierto de tiempo, de nuevo, la acción de los colectivos del barrio logró que el consistorio convocara a las asociaciones vecinales para negociar un uso provisional del solar mientras este permaneciera vacío con la condición de destinarlo a la dinamización de actividades sociales en el barrio. Como resultado de las distintas reuniones, en abril de 2015, una de estas asociaciones, el colectivo por la Masoveria Urbana per la Llar Alternativa (MULA), tomó la responsabilidad de abrir el espacio y gestionarlo bajo la auditoría de un grupo motor formado por el resto de asociaciones que acudieron a la reunión y del propio ayuntamiento. Los principales proyectos que propusieron fueron la creación de un huerto urbano con su propio sistema de compostaje, al que Ilamaron 'Quirhort', y la dinamización de actividades culturales para cohesionar el tejido social del barrio.

Respecto al huerto este consta de un conjunto de mesas de cultivo construidas por los vecinos y los cooperantes, así como de un sistema de compostaje que se dedica a reciclar la fracción orgánica que traen los vecinos y los sobrantes de fruta y verdura que ellos mismos recogen en las verdulerías cercanas. Con el compost generado, mezclado con fibra de coco, obtienen el sustrato para las jardineras. Este tipo de cultivo se adoptó debido a que el suelo de Espai Quiró estaba compuesto por una capa de runa y material de demolición triturado que no era adecuado para el cultivo. En el huerto se plantan de forma comunitaria productos de temporada que se reparten entre los vecinos que colaboran en las tareas de riego y de mantenimiento del huerto y la compostadora. 
Por otra parte las actividades lúdico-educativas que se llevan a cabo en el Espai Quiró incluyen los pases de "cine km 0" donde se proyectan documentales y películas producidas por colectivos o realizadores de los barrios adyacentes o que traten temáticas relacionadas con los mismos y con los movimientos vecinales. Junto a esto se hace una jornada mensual de puertas abiertas en la que se explica el proyecto in situ y se comparte también un picnic con productos del huerto y aportaciones de los vecinos. Asimismo, se hacen talleres y actividades con asociaciones del barrio.

\section{Colectivos implicados en el proyecto}

En cuanto al colectivo dinamizador del proyecto Espai Quiró hablaremos en primer lugar de la asociación que se hizo cargo del mismo, el colectivo MULA. Este, tal como explica su Web, inició su andadura en 2012 con un proyecto de masovería urbana en el barrio; concretamente se les cedió el permiso de habitar el Chalé Mercedes a cambio de realizar unas reformas estructurales en esta vivienda, de su cuidado y mantenimiento, así como de hacer un uso social del espacio desarrollando actividades con y para el barrio. Junto a los integrantes del colectivo colaboraron arquitectos, profesionales de la obra, abogados, así como vecinos y amigos que simplemente vinieron a ayudar. Una vez solventada la habitabilidad de su sede se fueron implicando en proyectos de mejora del barrio y en el refuerzo del tejido asociativo del mismo. Uno de estos proyectos es Espai Quiró en el que participan activamente algunos miembros del colectivo.

Aunado a ellos encontramos a uno de los fundadores del colectivo "Re:farm The City", quienes se definen en su página wiki como un laboratorio de personas y herramientas que ponen en práctica una manera diferente de vivir en las ciudades con ritmos más pausados, sostenibles y conscientes. Es un proyecto de experimentación por una vida más orgánica en el sentido más 
amplio y cuya aportación al proyecto está principalmente centrada en la construcción colectiva de las dinámicas comunitarias, así como de los procesos y herramientas para llevar a la práctica el huerto urbano y los equipamientos auxiliares.

También debemos mencionar al grupo de vecinos "Plataforma recuperemos la Plaza Sanllehy", los cuales se habían agrupado en una plataforma para la recuperación y ajardinamiento de esta plaza del barrio. Algunos de sus miembros siguen implicados en el Espai Quiró y forman parte del equipo motor, además de participar en otras reivindicaciones vecinales.

Finalmente hay que destacar el papel de diversas entidades colaboradoras y de algunos vecinos, los cuales han ido aportando sus varios conocimientos o su dedicación ya sea de forma esporádica o regular. Todo esto pretende reforzar el tejido asociativo del barrio y su red de contactos con otros actores sociales.

\section{Presentación del proyecto pedagógico de diseño de comunicación participativo}

Como hemos comentado, este estudio es una investigación-acción participativa (IAP) en la que investigamos a través del análisis de nuestra propia experiencia como participantes de una acción de codiseño entre los estudiantes y los miembros de la comunidad vecinal del Espai Quiró y el grupo de estudiantes de la ESDAP. Desde el punto de vista metodológico partimos de varios principios acordes con la idea de IAP (Pestaña y Alcázar, 2009). Por su parte el problema a estudiar había de surgir de la propia población implicada (Merino y Raya, 1993), además se debía establecer una negociación entre los actores implicados para definir qué acciones se iban a llevar a cabo y de qué manera (de Miguel, 1993); este hecho se llevó a cabo a través de reuniones y sesiones de lluvia de ideas dentro del marco de las asambleas vecinales. Por otra parte nos planteamos desarrollar y evaluar lo realizado con una doble finalidad: 
(i) facilitar información para otros sobre hechos concretos (Park, 1992) y (ii) conseguir que la información este constantemente circulando y en todas las direcciones. Para ello se pidió a los alumnos que llevaran un registro de todas las fases del proyecto, que se recogió en un blog, y que la información se compartiera a través de ese mismo blog para hacer aportaciones tanto los alumnos como el colectivo vecinal; además, se establecieron reuniones presenciales de trabajo y análisis conjunto a lo largo del proceso de diseño y producción. De esta manera se "trata de vincular la investigación a la acción, el conocimiento y la práctica para crear las soluciones operativas que necesitan los sectores populares" (Villasante, 1994, p. 424). Esta actividad se concretó en un proyecto de diseño de comunicación participativo entre la comunidad vecinal y los alumnos en los términos que relataremos a continuación.

\section{El grupo de estudiantes}

El grupo de alumnos lo componían 17 estudiantes de estudios superiores en diseño gráfico del Campus "L'Hospitalet. Serra i Abella" de la ESDAP. Más concretamente eran alumnos de la asignatura "Organización y procesos del diseño gráfico", del año académico 2016-17. Esta asignatura se imparte en el $7^{\circ}$ semestre del total de 8 que componen los estudios, por lo tanto este proyecto se llevó a cabo entre los meses de septiembre de 2016 y febrero de 2017. A nivel demográfico el grupo de estudiantes se componía de 11 alumnas $(64,7 \%)$ y 6 alumnos $(35,3 \%)$, con edades comprendidas entre los 21 y 27 años en el momento de iniciar el proyecto académico. De ellos 6 de 21 años (35,3\%), 0 de 22 (0\%), 5 de 23 (29,4\%), 1 de 24 (5,9\%), 2 de $25(11,8 \%), 1$ de 26 (5,9\%) y 2 de 27 (11,8\%), es decir que un 70,6 \% eran menores de 25 años y un 29,4\% estaba entre los 25 y por debajo de los 30. En este sentido podemos decir que se trata de un grupo bastante representativo de los estudiantes universitarios españoles si lo comparamos con la media española de finalización de los estudios de grado en todo el ámbito de las artes 
y humanidades en el año académico anterior (Figura 3), que se situaba en un $61,1 \%$ de mujeres respecto al $38,9 \%$ de hombres del total del alumnado en el que un $64 \%$ tenían menos de 25 años y un 17,9\% entre 25 y 30 años.
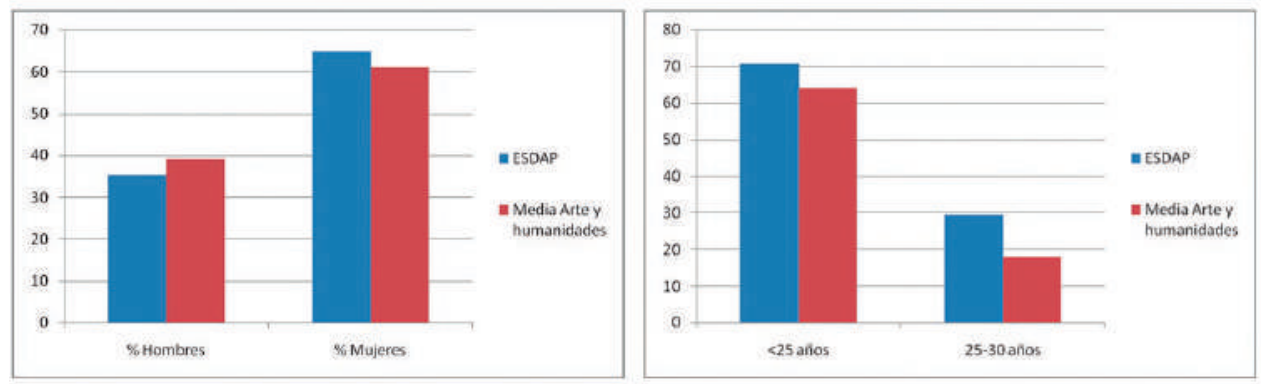

Figura 3. Figura comparativa del porcentaje de alumnos de la ESDAP de último curso participantes en esta actividad respecto a la media de alumnos que finalizaron los estudios en el área de artes y humanidades, según datos de la OCDE de 2015, segmentados por género y por edad. Fuente: elaboración propia por parte del autor.

Objetivos de aprendizaje, criterios metodológicos y premisas pedagógicas

En el plan docente de la asignatura "Organización y procesos del diseño gráfico" se demanda la adquisición de competencias tales como aprender a organizar y planificar el trabajo de forma eficiente y motivadora, integrarse adecuadamente en equipos multidisciplinares y en contextos culturales diversos, liderar y/o coordinar equipos de trabajo, conocer diversos procesos y materiales, coordinar la propia intervención con la de otros implicados y analizar, evaluar y verificar la viabilidad productiva de los proyectos.

En este sentido decidimos plantear una actividad académica en la que el alumnado tuviera que trabajar en un contexto de diseño participativo con un colectivo vecinal de composición diversa para aprender a organizar su trabajo de forma eficiente y motivadora no solo para sí mismos, sino también para 
los integrantes de un grupo de trabajo multidisciplinar y diverso; coordinando su propia intervención con la de los del proyecto, analizando y evaluando juntamente con ellos la viabilidad productiva del proyecto (ya que son estos, quienes mejor conocen las características y los recursos de su proyecto vecinal) y diseñando soluciones abiertas y escalables que los miembros de la comunidad pudieran ir ampliando o modificando en función de sus necesidades cambiantes.

Por otra parte, más allá de los objetivos estrictos de aprendizaje del plan docente, la idea concreta de poner en práctica nuestro aprendizaje en colaboración con una iniciativa de dinamización del espacio público en Barcelona fue una decisión motivada por la vigencia en el entorno del fuerte debate sobre el abuso de la propiedad inmobiliaria y la gentrificación en algunas zonas de la ciudad y el hecho de que esta sea pionera en la recuperación y dinamización de espacios vacíos para la regeneración de los barrios a través de planes de usos de solares vacíos como el Pla BUITS, así como el hecho de que la ciudad cuenta con una sólida red asociativa vecinal de larga tradición.

Respecto a los criterios metodológicos, diremos que para conseguir los objetivos de aprendizaje descritos fue idóneo aplicar dos criterios principales: el de la actuación local y el del diseño participativo con equipos multidisciplinares y soluciones abiertas. En cuanto al primero, permitía hacer un seguimiento cercano del desarrollo procesual al ser un proyecto local relativamente cercano a nuestra escuela en el que el alumnado podía visitar el espacio para intervenir y trabajar sobre el terreno. Y sobre el segundo, el diseño participativo se ajustaba a los objetivos pedagógicos de la organización del trabajo propio y de otros de forma colaborativa. Igualmente nos pareció idónea la comunidad del Espai Quiró debido a que, además de ser un proyecto vecinal local, el diseño participativo se ajustaba a su dinámica de grupo autogestionado y asambleario; esto nos brindó la oportunidad de debatir las ideas conjuntamente 
en sus reuniones, aceptando con entusiasmo participar tanto del diseño como de la producción de las intervenciones que se plantearan.

Todo esto permitió aplicar un método participativo en la definición de los diseños junto a la red de actores implicados, que componían un grupo multidisciplinar con aportes de personas voluntarias con diferentes background y habilidades, estableciendo equipos que permitieron aprovechar los diversos talentos tanto de los alumnos como de los miembros de la comunidad del Espai Quiró; asimismo, sus necesidades cambiantes y su idiosincrasia de comunidad orgánica encajaban perfectamente con la idea de generar soluciones abiertas que ellos mismos pudieran ir adaptando con el tiempo.

Desde el punto de vista de las premisas del proyecto, y en cuanto al criterio de actuar localmente, nos basamos en la premisa de que la acción sobre una problemática relativa a un entorno cercano y asequible al estudiante permite estudiarla en vivo y comprenderla con mayor profundidad. Esto supone dirigir la mirada del diseño hacia el compromiso con los esfuerzos de la sociedad civil por mejorar su entorno: colaborando en un proyecto autogestionado, enfocado, tal como el propio colectivo lo define, a la creación de tejido social y al empoderamiento de las comunidades vecinales en la gestión de su ocio, su alimentación y su espacio público, ofreciendo nuevos modos de habitarlo y promoviendo la implicación del usuario como agente fundamental en el diseño de su entorno.

Con base en el segundo criterio, el de establecer una forma de diseño participativa organizando equipos de trabajo multidisciplinares e implicando a los actores locales en la toma de decisiones de forma conjunta y diseñando soluciones abiertas y sostenibles, partimos de las conclusiones del Icograda Design Education Manifesto 2011 para la enseñanza del diseño en cuanto al codiseño participativo y abierto (Bennett and Vulpinari, 2011) donde se recomiendan 
las dinámicas de trabajo que promueven una participación continua a través de mecanismos para codiseñar en múltiples estados del trabajo y que dichos mecanismos se articulen alrededor de un proyecto lo suficientemente flexible para revisitar y hacer una reflexión crítica de los resultados de forma cíclica a lo largo de todo el proceso, especialmente en las fases finales cuando la apariencia de compleción de los prototipos pueda conducir a centrarse solo en los detalles y olvidar la idea general (Spinuzzi, 2005) para que el diseño tenga una incidencia real de transformación social.

También seguimos la recomendación de un diseño abierto en el que las soluciones pueden ser escalables, modulares, y desarrolladas por la propia comunidad (Raasch, Herstatt and Balka, 2009), de forma que sean los propios usuarios e implicados los que puedan ampliarlas o adaptarlas a sus necesidades a lo largo del tiempo. Finalmente se tomo en consideración las recomendaciones del diseño para la transición respecto a la aportación del talento de equipos multidisciplinarios a fin y efecto de favorecer el cambio hacia un modelo económico, productivo y de consumo más sostenible (Irwin, 2015). De ahí que no solo buscamos la diversidad de habilidades en la confección de los grupos de alumnos, sino que pedimos la colaboración de los miembros del colectivo para que aportaran sus conocimientos y destrezas en los grupos de trabajo donde consideraran que podían participar para encontrar soluciones innovadoras o para llevar a cabo los procesos de construcción y producción.

Con todo ello en mente, establecimos como segunda premisa el que la mejor forma de encajar el trabajo de nuestros alumnos en el esquema asambleario de la comunidad del Espai Quiró sería abordar el proyecto de diseño como un agente consultor dentro de la estructura horizontal de decisión y actuación del colectivo de voluntarios. De esta forma se planteó un proceso de codiseño participativo en el que tanto los encuentros asamblearios con los vecinos como los comentarios sobre las propuestas que se hacían en el 
blog del proyecto permitían comentar con los miembros del colectivo todo el proceso de conceptualización e implementación de soluciones de diseño en una cooperación no jerárquica sino en igualdad de condiciones, incluso dejando las soluciones abiertas de manera que los alumnos proporcionaran marcos de intervención que pudieran ir adaptándose y concretándose con su uso y con el paso del tiempo; además se tuvo en cuenta el uso de materiales de bajo impacto ambiental y estrategias de producción sostenibles como el upcycling (reutilizar productos usados y desechados para darles una nueva vida transformándolos en otros artefactos).

\section{Estructura del proceso proyectual para el desarrollo de las propuestas}

Primera fase. Toma de contacto, consenso en los objetivos del proyecto y términos del convenio de colaboración

En un primer contacto con la comunidad del Espai Quiró, en una de sus asambleas periódicas de septiembre de 2016, el profesor de la asignatura planteó como representante de la escuela la posibilidad de que los alumnos colaboraran con ellos en algún aspecto relacionado con el desarrollo de su proyecto comunitario; siempre dentro del marco de dicha asignatura y programando una actividad de aprendizaje específica para ese propósito. En esa asamblea se consideró interesante la propuesta en términos generales y se aprobó trabajar de manera conjunta y participativa, así como convocar al colectivo a una nueva asamblea para debatir sobre el contenido concreto de esta colaboración.

En la segunda asamblea tuvimos en cuenta que su red de voluntarios ya incluía arquitectos e ingenieros que se ocupaban del diseño de las instalaciones y mobiliario del huerto, y que nuestros alumnos cursaban un grado especializado en diseño gráfico. Considerando todo esto se llegó al consenso de que las 
necesidades del proyecto en las que mejor podríamos colaborar se podían agrupar en un proyecto de diseño gráfico de comunicación externa del proyecto dirigida a los vecinos, ya que el colectivo había detectado ciertos problemas de comunicación con el vecindario y los visitantes del espacio.

En concreto se manifestó que habían percibido que muchos vecinos pasaban junto al solar sin entrar y que si lo hacían, lo primero que preguntaban era que quién estaba ocupando el espacio y con qué finalidad. De hecho, su percepción era que los vecinos no conocían el proyecto del Espai Quiró y que incluso recelaban del uso que se le estaba dando al espacio por puro desconocimiento. Así pues, se decidió diseñar un proyecto de comunicación orientado a solventar estos problemas.

Por otra parte, en cuanto a la interacción entre la escuela y el colectivo del huerto, nos dimos cuenta de que un sistema de codiseño totalmente presencial se hacía inviable dado que el colectivo se reunía con una frecuencia mensual entretanto los alumnos disponían solo de unos tres meses para concluir el proyecto repartidos en solo 10 sesiones presenciales de 90 minutos en el aula y otras tantas horas de dedicación autónoma por parte del alumnado con la posibilidad de tutorías. Todo ello nos llevó a acordar una combinación de trabajo por separado de la comunidad del huerto y de los alumnos, más unas sesiones de trabajo conjuntas y una comunicación virtual entre ambos colectivos a través de un blog de acceso conjunto.

\section{Visita al huerto y reconocimiento del espacio}

La primera de estas sesiones presenciales fue una visita al Espai Quiró guiada por miembros del colectivo. En ella se explicaron las características del proyecto vecinal, del propio espacio (Figura 4), y de la comunidad del huerto. En el reconocimiento del espacio se detectó que solo existían unas 
pocas piezas de mobiliario que marcaban los usos del mismo. En concreto, nos mostraron: una pantalla de proyección situada frente a unas gradas hechas con palés de obra que definían la zona para la presentación de documentales y películas (Figura 5A); unas mesas, bancos y sillas realizadas con restos de obra y muebles recuperados que delimitaban el espacio de comedor y reunión (Figura 5B); una caseta realizada con palés y restos de obra que servía como zona de cocina y almacén, junto a unos contenedores de basura cedidos por el ayuntamiento que servían para recoger la fracción orgánica y realizar el proceso de compostaje (Figura 5C) y unas jardineras hechas con palés y un remolque que definían el espacio de cultivo del huerto con su propia zona de almacenaje (Figura 5D). Además los cooperantes comentaron con los alumnos los pormenores de su sistema de compostaje, el funcionamiento del huerto y la relación de las actividades que habían Ilevado a cabo hasta la fecha.

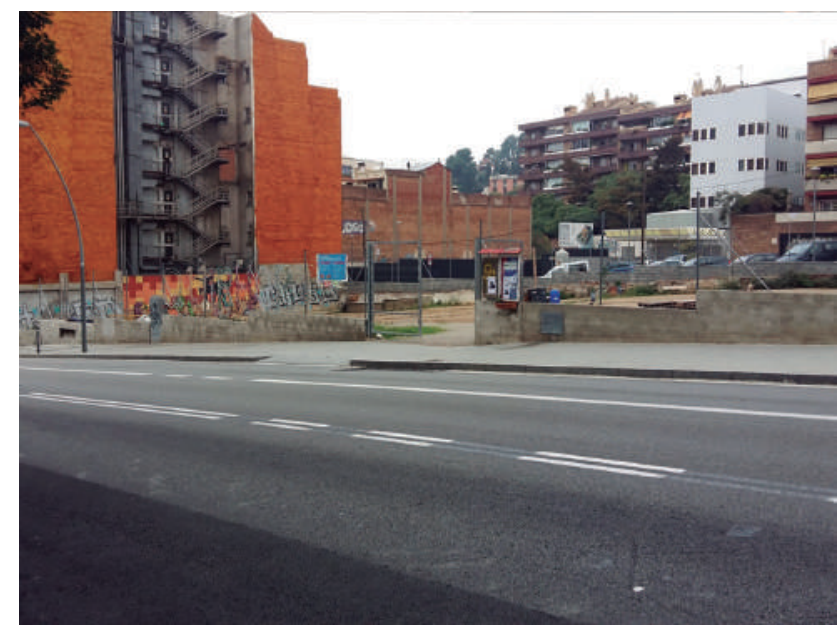

Figura 4. Vista panorámica del Espai Quiró antes de la intervención de los alumnos. Fuente: fotografía Nicola Scandogrlio. 


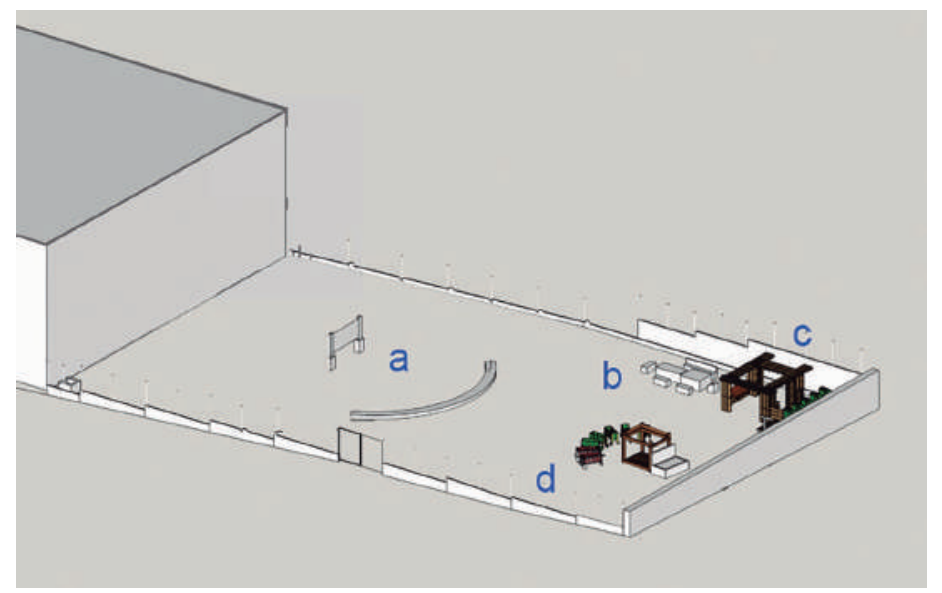

Figura 5. Esquema de la distribución del Espai Quiró antes de la intervención de los alumnos. Fuente: https://redissenyespaiquiro.wordpress.com/2017/01/25/resultat-

A partir de esta visita, y debatiendo lo observado, se llegó a la conclusión de que las grandes dimensiones y del espacio hacían muy difícil la percepción unitaria del mismo y de las actividades que se llevan a cabo en él; y que las piezas de mobiliario y construcción al interior del solar eran demasiado aisladas y pequeñas y no conseguían dar una percepción clara del espacio ni de su uso, con el agravante de la carencia de un presupuesto específico para su adecuación. A su vez, que las razones más probables para la falta de información de los vecinos eran básicamente las siguientes: que el espacio no estaba correctamente señalizado desde el exterior; que no existía ningún elemento informativo de las actividades que se hacían en él ni de la comunidad que gestionaba el proyecto; que tampoco había horarios claros de apertura al público ni un calendario de actividades visible desde el exterior; que solamente 
existía una pequeña banderola para explicar que se estaba haciendo un uso provisional del espacio a la espera de la ejecución del nuevo plan de usos municipal. Por último, también se apuntó que existía un déficit de herramientas pedagógicas para explicar los procesos de compostaje y agricultura urbana a los grupos escolares y vecinos que visitan este espacio.

Con esto se concluyó que era necesario mejorar la percepción del espacio, la información sobre su uso, estimular la participación entre los vecinos y transeúntes, así como diseñar unas herramientas pedagógicas para ser usadas con los escolares y otros visitantes.

\section{El proceso de definición, desarrollo e implementación de las propuestas de diseño}

La siguiente fase alternó el trabajo conjunto y por separado. Dentro de ella se realizó, en primer lugar, una lluvia de ideas entre los alumnos en el aula para pensar posibles actuaciones concretas que satisficieran las necesidades de comunicación. En segundo lugar, el colectivo Espai Quiró hizo una sesión de post-it brainstorming dentro de una de sus asambleas para pensar sus propias propuestas (Figura 6). A esta reunión asistió el profesor para dinamizar y asesorar en la sesión de lluvia de ideas y también para exponer las ideas de los alumnos a la comunidad y que estas se pudieran debatir y generar feedback. Toda la información de esta reunión llegó al grupo de alumnos a través de los post-it o tarjetas de sugerencias rellenadas por los miembros del colectivo (Figura 7) y del acta con las respectivas conclusiones sobre el trabajo de los alumnos en la asamblea. 


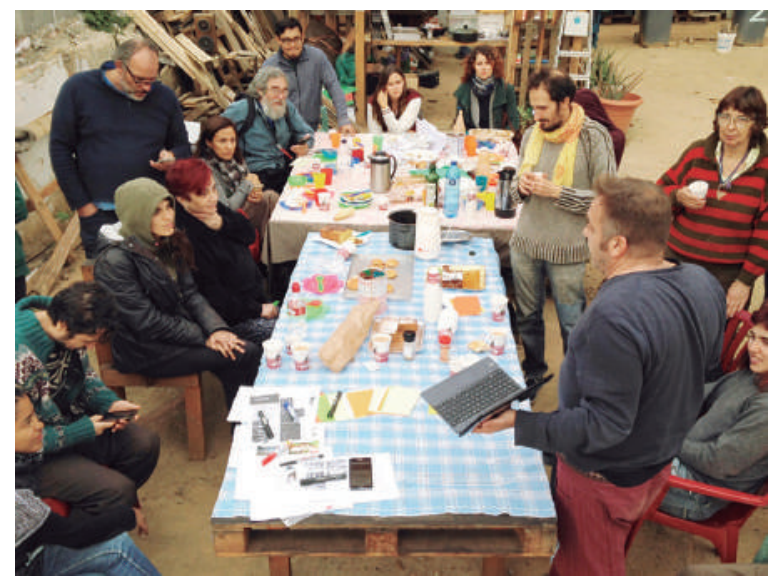

Figura 6. Sesión de post-it brainstorming con algunos miembros de la comunidad del Espai Quiró en una de sus asambleas periódicas. Fuente: fotografía Nicola Scandogrlio.

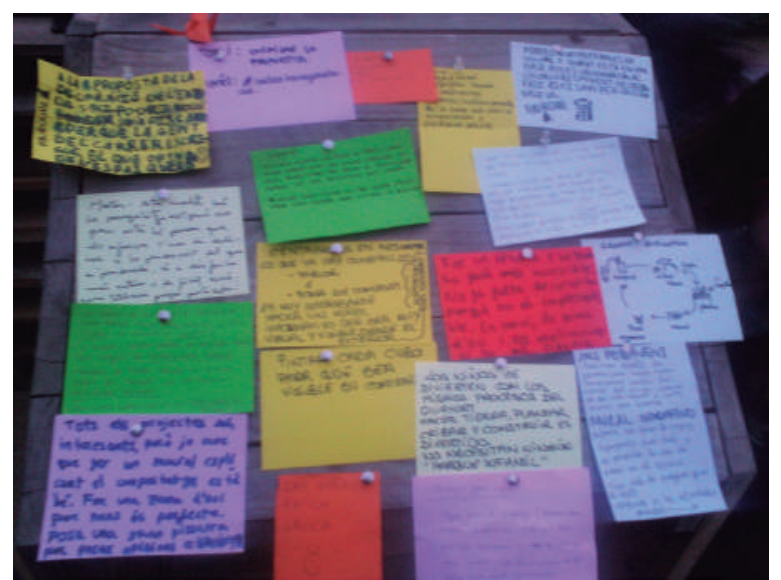

Figura 7. Resultados de la sesión de post-it brainstorming. Fuente: fotografía Nicola Scandogrlio. 
Posterior a esto, durante el desarrollo y concreción de las propuestas definitivas, se fueron sumando otros comentarios en el blog del proyecto de diseño de comunicación del Espai Quiró — realizado por los alumnos de la asignatura-. En este, los alumnos mantenían al día el estado de los avances del diseño para que tanto ellos como la comunidad vecinal pudieran discutirlos. Ocasionalmente también se comunicaron las ideas a través de correos electrónicos. Desde este momento, los alumnos desarrollaron sus propuestas publicando periódicamente sus avances en el blog. Posteriormente los miembros de la comunidad consultaban y exponían sus sugerencias, las cuales servían de base para el debate en el aula.

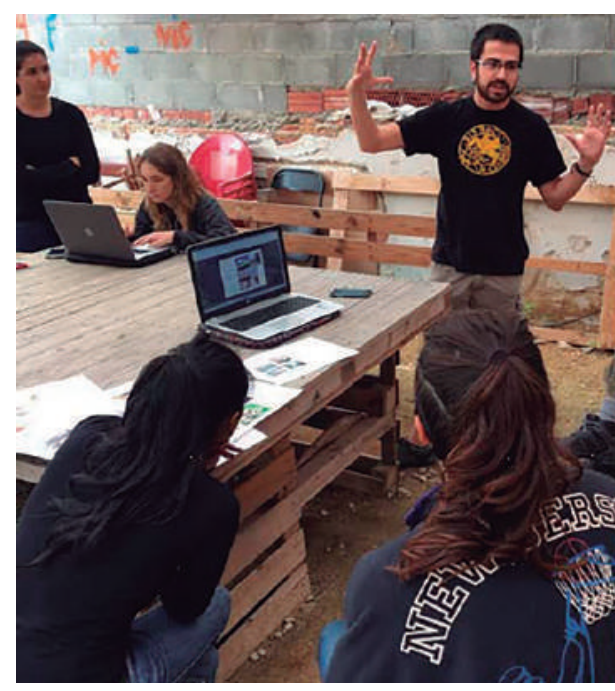

Figura 8. Los alumnos exponen sus propuestas a los miembros de la comunidad. Fuente: fotografía Nicola Scandogrlio. 
A pocas semanas del fin del ejercicio se hizo la segunda sesión conjunta presencial en el huerto, que consistió en una exposición de los proyectos por parte de los alumnos y un debate con la comunidad sobre sus detalles y la forma de organizarse para llevar a cabo su producción e implementación (Figuras 8, 9); luego se realizó un trabajo de preproducción en el aula para detallar todos los materiales y técnicas que se iban a emplear y el calendario de trabajo.

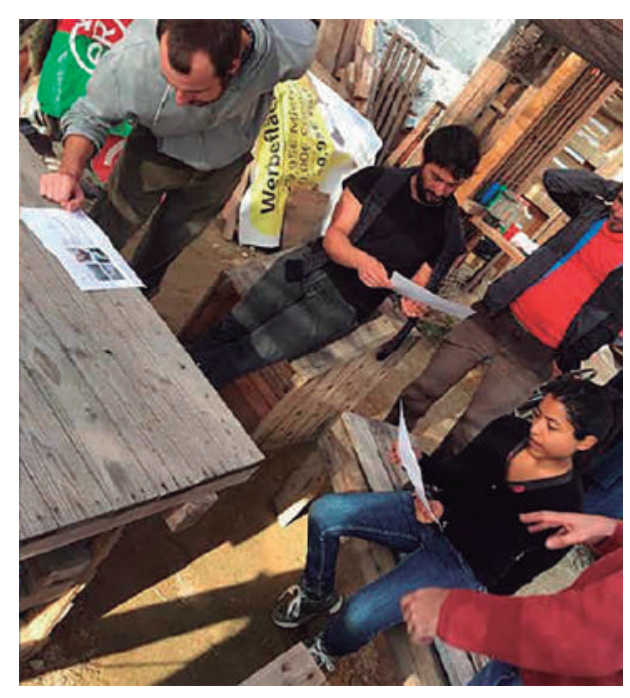

Figura 9. La comunidad analiza las propuestas. Fuente: fotografia alumnos de la escuela.

Por último, entramos en la fase de producción e implementación que incluyó la tercera y última sesión presencial de los alumnos en el huerto. Esta consistió en una jornada de trabajo conjunto en la que los alumnos y los miembros de la comunidad vecinal implementaron las intervenciones de forma colaborativa. 
La fase de producción contempló estrategias de upcycling como la reutilización de palés de obra usados para convertirlos en piezas de mobiliario entre ellos las jardineras del huerto y la bancada de asientos para el cine; el uso de materiales de bajo impacto ambiental y la combinación de herramientas y técnicas tradicionales con tecnologías digitales, las cuales se pudieron usar debido a la suma de habilidades y herramientas de los alumnos y los miembros de la comunidad. Estas intervenciones trataron de cumplir los objetivos, generando soluciones abiertas y escalables cuya concreción no acababa con la intervención de los alumnos sino que debía continuar de manera orgánica con el trabajo colectivo de la comunidad del huerto al ampliar y adecuar las soluciones a las necesidades cambiantes del Espai Quiró.

\section{Detalle de las propuestas realizadas}

A partir de las necesidades identificadas se proyectaron una serie de actuaciones que podemos agrupar alrededor de dos objetivos de comunicación principales: (i) la identificación y correcta lectura del espacio y del proyecto y (ii) la creación de materiales pedagógicos para informar a los grupos de escolares y visitantes del huerto.

\section{Identificación del espacio y del proyecto}

Así pues, las actuaciones referidas al primero de los objetivos se centraron en identificar el espacio y dar a conocer el proyecto Espai Quiró a los vecinos y transeúntes que observan el solar desde el exterior sin llegar a entrar ya sea porque encuentran el recinto cerrado o porque no se deciden a preguntar. Para disipar sus posibles dudas y recelos se propusieron las siguientes intervenciones complementarias: la primera fue pintar en una de las paredes medianeras un rótulo de 6 metros de ancho por 2,5 metros de alto, visible desde la calle, con el nombre del espacio y la descripción de sus actividades principales (Figura 10). 
La siguiente fue tejer el nombre del espacio en la valla trasera, complementando otro rótulo que ya habían tejido tiempo atrás los vecinos en la valla frontal de la avenida Verge de Montserrat. Esta última intervención se llevó a cabo con un proceso de upcycling, reutilizando banderolas promocionales de lona que se cortaban en tiras y se urdían en el tejido metálico de la valla exterior (Figura 10).

En tercer lugar se diseñó un mural con fotografías y textos que explicaban a los transeúntes las actividades que se llevan a cabo en el espacio. En este caso se reutilizaron planchas de madera de desecho para construir un mural modular de círculos de madera fijados a la valla metálica con alambre, a los que se adherían los textos y fotografías protegidos de la intemperie con barniz al agua para exteriores (Figura 10).

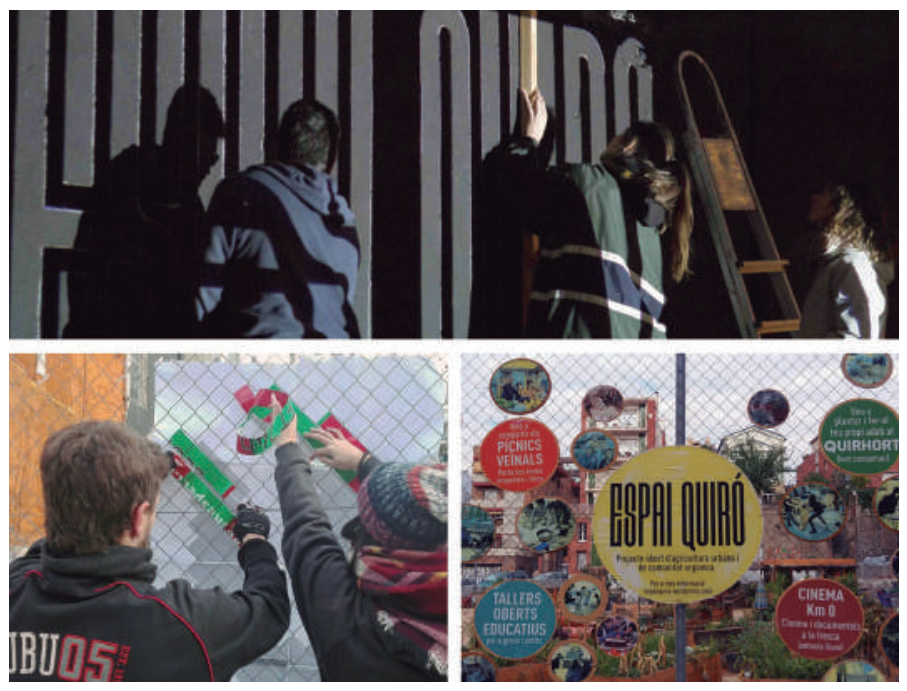

Figura 10. Alumnos pintando el rótulo identificativo de la pared medianera; alumnos tejiendo el rótulo identificativo de la valla trasera; mural modular de la valla de la entrada. Fuente: fotografia alumnos de la escuela. 
Finalmente para cohesionar el espacio se propuso ordenar y trasladar los materiales de construcción que se acumulaban en el huerto fuera de esta zona habitada, al igual que la aplicación de una gama cromática y algunos elementos de rotulación manual a los diferentes elementos de mobiliario para facilitar la coherencia visual del conjunto. La aplicación de la nueva gama cromática la iniciaron los propios alumnos y fue continuada por los miembros del colectivo vecinal utilizando pinturas con base de agua para evitar el uso de disolventes químicos (Figura 11).
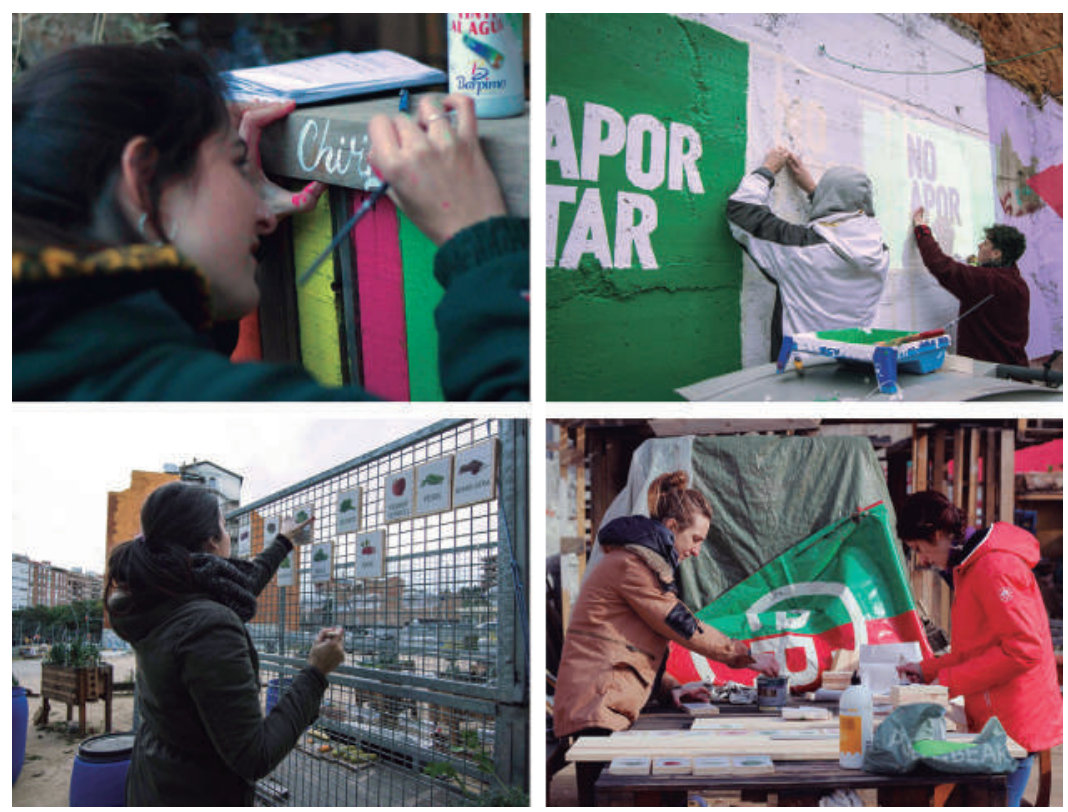

Figura 11. Alumna aplicando rotulación y la gama cromática a los elementos de mobiliario; alumnos pintando el mural educativo de la zona de compostaje; alumna montando las piezas del calendario de la huerta sobre su soporte; alumnas en el proceso de trabajo del calendario de la huerta. 


\section{Diseño de materiales pedagógicos}

Para informar a los grupos de escolares de primaria y secundaria que venían a conocer el huerto y a los visitantes ocasionales que solicitaban información in situ se pintó un mural explicativo sobre el proceso de compostaje en el huerto (Figura 11) y se diseñó un calendario del cultivo de los productos de temporada apropiados para la producción local en nuestro clima (Figura 11). Ambos se diseñaron para utilizarlos como herramientas formativas en las visitas guiadas al Espai Quiró.

\section{Resultados del proceso de codiseño con entidades locales en equipos multidisciplinares y con soluciones abiertas}

A partir de las premisas del proyecto y del desarrollo del mismo se obtuvieron una serie de resultados de aprendizaje que comentaremos a continuación.

\section{Trabajo local con comunidades vecinales y seguimiento cercano del proceso}

El primero de ellos se refiere a que el uso de un proceso participativo de codiseño con los actores locales permitió a los alumnos conocer mejor y de primera mano el proyecto vecinal, sus actores y su problemática, así como trabajar sobre el terreno de manera conjunta con una visión más realista y compartida del mismo.

En este sentido, si bien no se pudo llevar a cabo un trabajo totalmente presencial de los alumnos en el espacio a intervenir debido a cuestiones de horarios académicos antes mencionadas, se pudo conseguir una cercanía entre ambos colectivos a través de la mencionada combinación de sesiones presenciales y comunicación telemática. De igual manera algunos de los alumnos acabaron 
por acordar alguna sesión presencial extraordinaria, encontrándose de forma autónoma con los miembros del colectivo usando parte de sus horas de trabajo autónomo y tutorado contempladas en los créditos ECTS de la asignatura.

\section{Diseño y toma de decisiones participativa}

Las aportaciones de los miembros del colectivo y la capacidad de adaptación de los alumnos fueron claves para conducir el proyecto hacia una solución comprometida con las necesidades reales del mismo desde el principio y a lo largo de todo el proceso. Prueba de ello es que desde el inicio del proceso se pudieron reorientar algunas propuestas.

En concreto, una de las propuestas que se transformaron a través del debate comenzó con la idea inicial de los alumnos de diseñar una zona de juegos para los hijos de los voluntarios y vecinos. Sin embargo en la asamblea comunitaria se argumentó que desde el punto de vista pedagógico parecía más interesante que los niños jugaran de una forma más libre, creativa y constructivista, incluyéndolos en la propia dinámica del espacio, colaborando si querían con los adultos o creando sus propios juegos y juguetes con los materiales del huerto en lugar de habilitarles una zona artificial de juegos. Esto llevó a reorientar la propuesta antes de emplear un tiempo muy valioso en su desarrollo.

Otro ejemplo, esta vez en la fase de desarrollo de las propuestas aceptadas, es el mural que se construyó en la valla exterior del recinto. Los alumnos habían planteado inicialmente imprimir una lona explicativa y fijarla en el exterior del recinto, pero se llegó a la conclusión de que era más interesante realizar el mural modular por 2 razones principales: (i) se prefería que el mural se diseñara y construyera de forma colaborativa entre los miembros del colectivo y los alumnos, uniendo los conocimientos y habilidades constructivas de unos y otros; (ii) de acuerdo a las premisas del diseño abierto se consideró más 
interesante que las piezas se pudieran sustituir o reposicionar en un futuro para modificar o ampliar la composición a medida que se hicieran nuevas actividades.

\section{Soluciones de diseño abiertas y escalables}

Sobre este aspecto del diseño abierto, comentaremos que una vez presentadas las propuestas e implementadas en una primera fase, los alumnos se habían asegurado de haber puesto las bases para que el propio colectivo de usuarios pudiera seguir desarrollándolas y haciéndolas crecer o evolucionar en función de sus necesidades cambiantes. De hecho, semanas después de terminar la colaboración con los alumnos, en la asamblea se decidió crear una comisión con un pequeño grupo de vecinos que quisieran continuar las tareas de mejora de la comunicación. Y precisamente, su primera actuación fue hacer una ampliación del mural con nuevas imágenes. Esto se hizo mediante una selección participativa en línea en la que los vecinos pudieron escoger las fotografías que mejor representaran, a su modo de ver, la trayectoria del Espai Quiró. Posteriormente la comisión ordenó e hizo una selección definitiva en papel de las más votadas para dar coherencia al conjunto, finalmente los vecinos dieron su parecer y se llevó a cabo la ampliación del mural (Figura 12).

Otro ejemplo de diseño abierto y del trabajo de esta comisión es la mejora del tablón de anuncios donde se publicitan las actividades que se van realizando periódicamente tales como la cartelera del cine $\mathrm{km} 0$ del mes en curso, la fecha de la próxima jornada de picnic orgánico y puertas abiertas y el resto de actividades sociales y culturales. Esta mejora ya se había apuntado en las propuestas de los alumnos, pero no se terminó de desarrollar y quedó latente. No obstante, transcurrido un tiempo, al colectivo le pareció interesante retomarla y la comisión se encargó de ello. Para hacerlo se aprovechó un marco de ventana reciclado que estaba en la entrada y se le añadieron unos 
listones pintados siguiendo la gama cromática del proyecto general, además de un rótulo de letras de madera realizadas con un plóter de corte láser en un laboratorio público de fabricación digital gestionado por el ayuntamiento de la ciudad. Este rótulo sirve para anunciar de forma genérica las actividades donde se cuelgan carteles con las actividades concretas de cada mes. Profundizando en la colaboración, muchos de estos carteles son diseñados hoy por los propios vecinos y miembros del colectivo (Figura 12). Además de las tareas de diseño, en todo el proceso de construcción tuvieron un papel relevante los miembros de la comunidad (Figura 12).
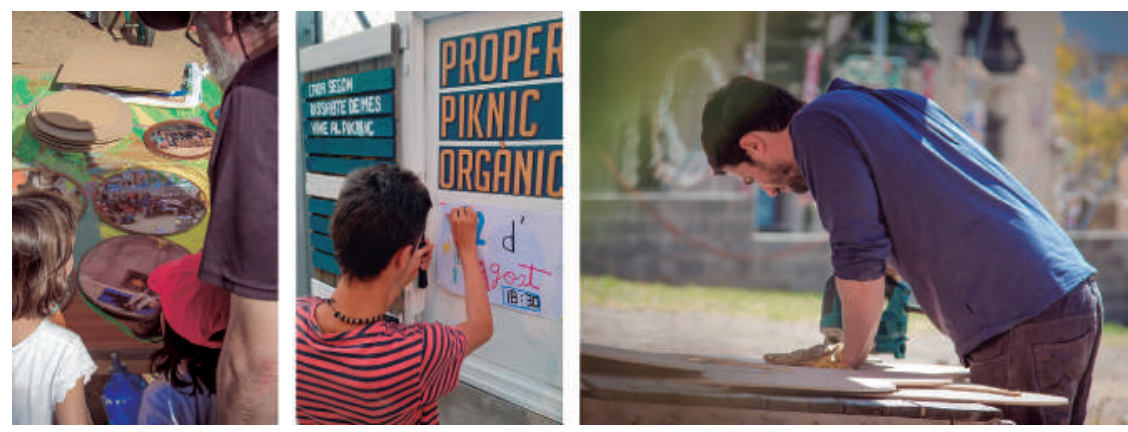

Figura 12. Niños y mayores revisan y dan su opinión de la selección de fotografías para colgar en el mural; un chico, vecino del huerto, cuelga un cartel realizado por el mismo en el tablón de anuncios; un miembro del colectivo MULA colabora en la construcción del mural de círculos de madera.

También queremos señalar un último ejemplo donde el papel de los usuarios fue incluso más significativo, se trata de la reordenación del espacio. En este caso se partía de la necesidad de mejorar la percepción del mismo y el conocimiento del proyecto de dinamización vecinal. Para ello se optó por agrupar las instalaciones en un área más reducida, ordenar los elementos de mobiliario e instalaciones y aplicarles un código cromático que les diera coherencia visual. 
En cuanto al resultado, si bien es cierto que estas intervenciones dieron una escala más humana y un aspecto más amable al conjunto, los alumnos por cuestiones de tiempo solo pudieron intervenir directamente en la primera fase de aplicación del nuevo cromatismo; quedando aún por resolver la parte más importante, la reordenación del espacio. Justamente, en este aspecto, el colectivo planteó un plan más ambicioso al que se había sugerido inicialmente: una nueva distribución en espiral del huerto (Figura 13) con varias nuevas mesas de cultivo mucho más grandes que las anteriores y dispuestas en la zona central del huerto, lo que permitió una mayor área de plantado y proporcionó una visión más ordenada desde el exterior (Figura 13). En esta ampliación tuvo un papel muy destacado Re-farm the city (Figura 13).

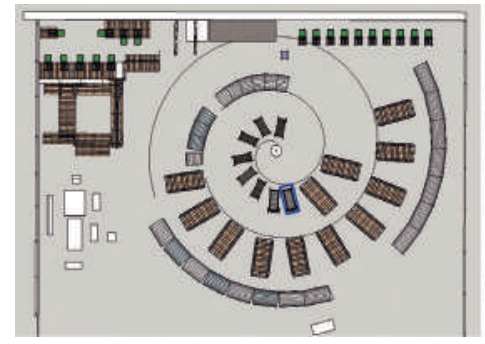

Figura 13. Vista detalle de la distribución en espiral de las nuevas jardineras; proyecto de ampliación del huerto realizado por la comisión vecinal; un miembro de Re-farm the city trabaja en la implementación de la nueva disposición en espiral de las jardineras diseñadas por él mismo. Plano: Hernani Días. Fotografias: Comunitat Espai Quiró

A estas actualizaciones y ampliaciones del diseño inicial han seguido otras que hacen que el proyecto continúe vivo y abierto. En definitiva, el trabajo en equipo de los alumnos de diseño junto a los actores implicados en la iniciativa vecinal ha permitido que los primeros pudieran poner en práctica y aprender una nueva forma de diseño cercana con visitas periódicas y contacto directo con la comunidad de usuarios; en donde más que proyectar y delinear unas soluciones terminadas se debatieron y consensuaron, en un proceso participativo 
de grupos multidisciplinares, unas propuestas que se materializaron en unos marcos abiertos de trabajo para que los usuarios pudieran desarrollarlos de forma escalable para crecer de forma autónoma en función de las necesidades cambiantes del colectivo y del proyecto.

\section{Conclusiones}

El proyecto pedagógico de diseño de comunicación del Espai Quiró se propuso partiendo de unas premisas pedagógicas de renovación de la enseñanza del diseño expresadas en documentos como el "The Icograda Design Education Manifesto" y criterios metodológicos vinculados a los principios del diseño para la transición. La motivación hacia este tipo de proyecto surgió de vincular objetivos de la asignatura "Organización y procesos del diseño gráfico" relacionados con la organización de un proceso de diseño participativo, colaborando con equipos multidisciplinares en el contexto local de las comunidades vecinales y con la idea de diseñar soluciones abiertas y escalables; pues consideramos que ello permitiría aprender a organizar su trabajo de forma eficiente y motivadora no solo para sí mismos sino para los integrantes de un grupo de trabajo multidisciplinar y diverso; coordinando su propia intervención con la de los usuarios y miembros del proyecto, analizando y evaluando con ellos la viabilidad productiva de los proyectos (ya que son estos, quienes mejor conocen las características y los recursos de su proyecto vecinal).

A lo largo del desarrollo de esta actividad pedagógica se han obtenido los resultados expuestos que suponen, a nuestro criterio, un interesante precedente en la forma de establecer la relación entre el trabajo de los estudiantes de diseño y las necesidades de la sociedad en la que se inserta su trabajo. De esta experiencia destacamos, a modo de conclusión, tres principios metodológicos que pueden ser de utilidad para futuras experiencias en el ámbito de la enseñanza del diseño. 
El primero de estos principios es el de favorecer el trabajo con comunidades locales, lo que ha supuesto un satisfactorio grado de adecuación de las propuestas de los alumnos a las necesidades de estos colectivos. Esto se atribuye al trabajo en un entorno accesible que facilita la acción sobre el terreno y un seguimiento cercano del proyecto, así como al trabajo con los actores implicados que juegan un papel fundamental para explicar de primera mano a los alumnos las condicionantes y particularidades del proyecto. De este modo se ha conseguido aprender a trabajar a través de un proceso de codiseño participativo.

En segundo lugar nos ha parecido útil el haber establecido equipos multidisciplinares de alumnos y actores locales, ya que permite aprovechar los diversos talentos del grupo para llegar a acometer entre todos la producción de las intervenciones. En este caso, las colaboraciones se llevaron a cabo usando materiales reciclados del propio entorno a partir de estrategias de upcycling y de reutilización que suponen un avance hacia formas más ecoeficientes y sostenibles de entender el diseño y su aprendizaje.

Finalmente ha sido muy enriquecedor trabajar con una metodología de diseño abierto dado que nos ha permitido plantear y crear soluciones escalables que los propios usuarios han ido modificando y ampliando en función de sus necesidades, logrando así un empoderamiento de la comunidad de vecinos del Espai Quiró en la toma de decisiones y hacer suyas las soluciones abiertas de diseño para continuarlas por sí mismos.

Sobre la respuesta de los estudiantes, estos manifestaron en los respectivos documentos del blog del proyecto diversas dificultades y retos a los que tuvieron que enfrentarse y que se transformaron a la vez en aprendizaje. Uno de los principales fue el hecho de adaptarse a diseñar de forma participativa, ya que algunas de las hipótesis que plantearon los estudiantes no encajaron 
inicialmente con la visión del colectivo del Espai Quiró debido a que no se había tenido en cuenta su idiosincrasia y forma de funcionamiento interno. Todo ello obligó a reemprender la búsqueda de nuevas alternativas una y otra vez con el consiguiente trabajo añadido y retraso en el calendario de trabajo.

Aunque este rechazo inicial de algunas de las propuestas generó una lógica frustración en el alumnado, también se consideró que esta experiencia de ensayo-error promovía un aprendizaje más claro y duradero; pues — si bien la toma de decisiones horizontal con los agentes implicados demanda una mayor tenacidad para llegar a acuerdos- tiende a generar unas soluciones mucho más adecuadas, personalizadas y satisfactorias para los usuarios, al igual que fortalece las habilidades de negociación y dirección de grupos.

Por otra parte fue muy interesante el proceso de producción conjunta en que los alumnos tuvieron una respuesta muy positiva ante la colaboración de los diversos miembros del colectivo que les apoyaron sobretodo en su conocimiento de los materiales y técnicas para los trabajos de carpintería, montaje de andamios para la pintura mural y demás tareas como el manejo de taladros y otras herramientas que ellos desconocían por completo. Esta forma de trabajar sumando habilidades en grupos heterogéneos fue sumamente enriquecedora para su aprendizaje en el ámbito de la producción y fomentando valores transversales como el trabajo en equipo, la organización de grupos de trabajo y la toma de decisiones por consenso. Finalmente fue notable la constatación, una vez terminado este proyecto, de cómo las soluciones aportadas fueron tomando envergadura y desarrollándose en función de las necesidades del colectivo de usuarios. 


\section{Referencias}

Barnbrook, J. et al. (1999). First Things First Manifesto 2000. Eye Magazine. Recuperado de http://www.eyemagazine.com/feature/article/first-thingsfirst-manifesto-2000.

Bennett, A. and Vulpinari, O. (Ed.). (2011). Icograda Design Education Manifesto 2011. Treviso, Italy: Grafiche Tintoretto.

de Miguel, F.M. (1993). La IAP un paradigma para el cambio social. Documentación Social, 92, 91-108.

Hopkins, R. (2008). The transition handbook: From oil dependency to local resilience. Cambridge, United Kingdom: UIT Cambridge LTD.

Irwin, T. (2015). Transition Design: A proposal for a new area of Design Practice, Study, and Research. Design and Culture, 7 (2), 229-246.

Irwin, T. et al. (2015). Transition Design. Recuperado de http://design.cmu.edu/ sites/default/files/Transition_Design_Monograph_final.pdf.

Merino, L. y Raya, E. (1993). El método de la investigación-acción participativa como mediación entre la teoría y la práctica de la formación del/a trabajador/a social y en el desarrollo profesional. En Seminario de integración teoría-práctica en la formación de los trabajadores sociales. EUTS de Alicante, Alicante, España.

Park, P. (1992). Qué es la investigación-acción participativa: perspectivas teóricas y metodológicas. En M.C. Salazar (Coord.), La investigación-acción participativa: inicios y desarrollos (pp. 135-174). Madrid, España: Editorial Popular.

Pestaña, M. y Alcázar, M.A. (2009). Investigación-acción participativa. En R. Reyes (Dir.), Diccionario crítico deficiencias sociales. Madrid, España: Plaza y Valdés, Universidad Complutense de Madrid. 
Raasch, C., Herstatt, C. and Balka, K. (2009). On the Open Design of Tangible Goods. R\&d Management, 39 (4), 382-393.

Seyfang, G. and Smith, A. (2007). Grassroots Innovations for Sustainable Development: Towards a New Research and Policy Agenda. Environmental Politics, 16, 584-603.

Spinuzzi, C. (2005). The Methodology of Participatory Design. Technical Communication, 52 (2), 163-174.

Villasante, T.R. (1994). De los movimientos sociales a las metodologías participativas. En J.M. Delgado y J. Gutiérrez (Coords.), Métodos y técnicas cualitativas de investigación en ciencias sociales. Madrid, España: Editorial Síntesis.

Como citar: Morales, J. (2019). Experiencias pedagógicas en el ámbito del diseño para la transición. Estudio de un caso: el proyecto de comunicación del Espai Quiró. Revista KEPES, 16 (19), 217-251. DOI: 10.17151/ kepes.2019.16.19.9 